\title{
La naturaleza de las naturalezas patrimonializadas. Una aproximación a las formas hegemónicas de representar lo natural ${ }^{1}$
}

\author{
Beatriz Santamarina Campos \\ Universidad de Valencia \\ Beatriz.Santamarina@uv.es
}

Resumen: En este artículo examinamos la forma de conceptualizar la naturaleza a través de las áreas protegidas (patrimonialización de la naturaleza). Desde el discurso hegemónico, la naturaleza — susceptible de ser «patrimonializada»— cumple una serie de requisitos que dibujan en el imaginario lo propiamente natural. Nuestro propósito es analizar cómo, a través de las categorías y las declaraciones efectuadas por los distintos organismos, se configura un prototipo ideal de lo que es la naturaleza. En las declaraciones para la protección de la naturaleza podemos encontrar un discurso tanto explícito como implícito de aquello que es leído y protegido como la natural naturaleza. De tal forma que, en los dispositivos de configuración y legitimación de los espacios naturales, podemos hallar un primer marco de análisis para acercarnos a los procesos políticos normalizados de asignación y distribución de autenticidad (verdad). O dicho de otra forma, los procesos de patrimonialización natural, sean internacionales, nacionales o locales, son una oportunidad para reflexionar sobre la dimensión política de lo natural. En este sentido, entendemos que el trabajo para la definición y el establecimiento de categorías de los espacios naturales es especialmente interesante, porque del mismo se desprenden importantes cuestiones ideológicas y prácticas.

Palabras clave: naturaleza; patrimonio; áreas protegidas; políticas hegemónicas.

1 Este trabajo se enmarca en el proyecto «El patrimonio cultural y natural en tiempos de crisis. Retos, adaptaciones y estrategias en contextos locales» (CSO2015-68611-R), financiado por el Ministerio de Economía y Competitividad. 
Abstract: The focus of this article is to analyze the conceptualization of nature within protected areas (patrimonialization of nature). From the viewpoint of the hegemonic discourse, nature fulfill the requirements needed to draw the contours of what is strictly speaking «natural» in social imaginaries. My main aim is to analyze how a specific and idealized prototype of what nature should be is configured through the categories and declarations of a variety of organizations. In many documents aiming to the protection of nature we could find both an explicit and implicit discourse referring to what is understood and protected as «natural nature». These documents configuring and legitimizing natural areas could be used as a first step to approach normalized political processes assigning or distributing authenticity (true). In other words, the patrimonialization of nature, being international, national or local, is an excellent opportunity to reflect on the political dimension of the 'natural'. In this sense, the work devote to define and establish categories in relation to natural areas is of the upmost importance since it can offer interesting insights into relevant ideological questions and practices.

Keywords: nature; heritage; protected areas; hegemonic policies.

\section{Introducción}

En este texto queremos abordar la particular forma de conceptualizar la naturaleza a través de las propuestas de protección de las áreas naturales protegidas (patrimonialización de la naturaleza). Desde el discurso hegemónico, la naturaleza - susceptible de ser «patrimonializada» - cumple una serie de requisitos que dibujan en el imaginario lo propiamente natural. A modo de cata, pretendemos analizar cómo, a través de las categorías y las declaraciones efectuadas tanto por los organismos internacionales, en concreto el Patrimonio Natural de la Humanidad declarado por la Unesco, como nacionales, en particular la figura de Parques Nacionales del Estado español, se configura o se ha venido configurando un prototipo ideal de lo que es la naturaleza $a^{2}$. Es decir, en las declaraciones efectuadas para la protección de la naturaleza podemos encontrar tanto un discurso explícito como implícito de aquello que es leído y

2 Prototipo, lógicamente, que ha ido variando a lo largo del tiempo y cuya transformación más significativa ha sido la inclusión de las Áreas Marinas Protegidas en los últimos años. 
protegido como la natural naturaleza. Y teniendo presente que las representaciones que manejamos en torno a lo natural determinan nuestra relación con ella, su comprensión se hace fundamental para entender cómo construimos la imagen de lo natural, y cómo la asignación de significaciones conlleva actitudes, valores y comportamientos que se generan frente a ella (Eder, 1996). Pero no solo nos interesa subrayar este aspecto, también se torna fundamental, como ya se ha señalado, reconocer que «descriptive categories end up having material effects» (West y Brockington, 2006: 610), al suponer una nueva regulación y reconfiguración de los territorios (simbólica, política, económica y jurídica) (Santamarina, 2009).

Desde esta perspectiva, se entenderá que los procesos de patrimonialización natural, sean internacionales, nacionales o locales, son una oportunidad para reflexionar sobre la dimensión política de lo natural, ya que dichos organismos, al realizar políticas para la conservación de la naturaleza, definen e instauran naturalezas en las áreas protegidas. O lo que es lo mismo, configuran y representan el universo de lo natural a partir de una insistencia en su pretendida o adjudicada naturalidad. En este sentido, entendemos que el trabajo para la definición y el establecimiento de categorías de los espacios naturales es especialmente interesante, porque de él se desprenden importantes cuestiones políticas (exportación y colonización de modelos cognitivos, geopolíticas patrimoniales) y prácticas (reglamentaciones de usos y recursos de áreas). Visto de este modo, la descripción y clasificación de las distintas áreas protegidas es un ejercicio ideológico que tiene innumerables consecuencias prácticas y discursivas.

En nuestra propuesta de análisis, a través de las declaratorias del Patrimonio Natural de la Humanidad y de los Parques Nacionales, se hace patente la instrucción y prescripción de patrones cognitivos. Por un lado, la agencia internacional de la Unesco propone lugares naturales, bajo la definición específica de ciertos criterios legitimados por su carácter tecnocientífico, dibujando un mapa de dónde, cómo y por qué se encuentra la naturaleza. En gran medida, el universo de lo natural dibujado por esta institución coincide con los parques nacionales declarados en los distintos países. Por otro lado, los parques nacionales representan el éxito del modelo decimonónico de protección estadounidense como paradigma oficial de la conservación de la biodiversidad. Modelo que, como ha sido ampliamente señalado, implicó una nueva regulación del territorio bajo un único patrón interpretativo, cimentándose en la contemplación 
de una pretendida wilderness, con el propósito de la salvaguardia de una natural naturaleza (Stevens, 1997; Diegues, 2005; West et alii, 2006; Selmi y Hirtzel, 2007; Adams y Hutton, 2007; Brockington, Duffy y Igoe, 2008; etcétera). La ansiada naturaleza salvaje se conformó como un escenario único, merecedor de la conservación para la contemplación, el estudio y el disfrute (Hutton, Adams y Murombedzi, 2005; Igoe, 2006; Selmi y Hirtzel, 2007; Descola, 2007) ${ }^{3}$ y como un productor notable de topografías patrióticas (Daniels, 1993).

Este paradigma conservacionista se extendió rápido a otros países ${ }^{4}$ y la construcción de una originaria naturaleza - la naturaleza prístina- pasó a tener un peso cada vez mayor en las agendas políticas conservacionistas. De esa forma, se ha acabado consolidando como una fórmula globalizada y naturalizada, basada en una pretendida verdad objetivada y amparada por la voluntad última y altruista - y no por ello menos importante - de proteger espacios para todos y para el futuro, frente a la voracidad y la expansión de nuestro sistema político-económico. Pero, si bien es cierto que la necesidad de proteger y conservar está fuera de toda duda, la imposición de una visión única en torno al mundo de lo natural, y por ende cultural, ha implicado el borrado de otras formas de entender las naturalezas y de relacionarse con ellas (Descola y Palsson, 1996; Hornborg y Pálsson, 2000; Escobar, 2000; etcétera) $)^{5}$.

Desde nuestra consideración, abordar la construcción de la naturaleza hegemónica, teniendo presente que la producción de la naturaleza es una realidad histórica (Smith, 2007: 39), permite atisbar los espacios naturales desde otras ópticas. Y nos acerca a la compresión de las áreas naturales como campos de poder, donde se reproducen las grandes narrativas de la modernidad, cimentadas en la constitución de categorías depuradas que actúan como confinadoras para la intervención de sujetos y objetos reificados (Latour, 1993; Foucault, 1970 y1991; Bourdieu, 1991 y 1998; Comaroff y Comaroff, 1991). En esta dirección, la naturaleza puede ser leída como un generador de autenticidad

3 Dejamos para otra ocasión las consecuencias políticas y económicas de este modelo. Para una aproximación se puede ver, entre otros: Runte (1977); Stevens (1997); Daniels (1993); Fortunato (2005); West et alii (2006).

4 Los primeros en seguir esta praxis fueron países con amplias extensiones de territorio (Canadá, Australia, Sudáfrica) (Nash, 1970; Solé y Bretón, 1986; Mulero, 2002; McNamee, 2010; Santamarina, Vaccaro y Beltran, 2014). El paradigma desembarcará en Europa a comienzos del xx: Suecia, Alemania, Suiza y España serán los primeros países en sumarse a la conservación pública y moderna inaugurada con Yellowstone.

5 Está de más reseñar que las distintas prácticas culturales dibujan una multiplicidad de naturalezas, mediadas y mediatizadas, a partir de una cosmovisión que intenta ordenar distintos mundos. 
(piedra angular de las activaciones patrimoniales), constituida a través del discurso hegemónico de la biodiversidad (Escobar, 1998; Holmes, 2011). Al mismo tiempo, esta posición permite destapar las particulares relaciones que entablamos con el medio, sacando a la luz la paradoja insostenible que detenta nuestro sistema: conservar y destruir al unísono.

Sin duda, hablar de naturaleza, o mejor dicho, hablar de naturalezas, es un ejercicio complejo en la medida en que esta es arbitrada, aunque se presente pretendidamente objetivada y sin intervención. El discurso de lo natural se hace vigente en la búsqueda de un quimérico estado de naturaleza representado, de forma magistral, en los espacios naturales. Existirían, pues, distintas naturalezas, o una gradación de las mismas que va desde la elaboración de una noble naturaleza (recreación mítica exigida) a la percepción de una naturaleza quebrantada (violación del estado pasado y perdido). En cualquier caso, delimitar la naturaleza, como reducto de lo natural, en las áreas protegidas garantizaría la natural naturaleza intemporal (Hutton, Adams y Murombedzi, 2005; Igoe, 2006; Selmi y Hirtzel, 2007). De esa forma, en los dispositivos desplegados de configuración y legitimación de los espacios naturales podemos hallar un primer marco de análisis para acercarnos a los procesos políticos normalizados de asignación y distribución de autenticidad ${ }^{6}$. Y recordemos que la autenticidad es una cualidad que certifica verdad (acreditación de lo verdadero), y la producción de verdad es un instrumento central de poder (Foucault, 1991; Haraway, 1995; Mignolo, 2012).

En suma, en este trabajo nos acercamos a la particular forma de conceptualizar la naturaleza a través de dos formas de patrimonialización natural, con el objeto de desvelar qué imaginario atraviesa el mundo de lo natural y cómo condiciona percepciones y actuaciones. Para ello, en primer lugar, nos acercamos a la configuración de las áreas naturales en España, con una pequeña contextualización para ubicar su producción. Pese a que solo contamos con quince parques nacionales, nuestro sistema de protección natural parece dibujar una idea clara de dónde está la naturaleza. En segundo lugar, nos aproximamos a los sitios declarados por la Unesco como Patrimonio Natural de la Humanidad. Detrás de sus declaratorias también es posible atisbar dónde se encuentra lo natural para esta instancia de normativa global. Por último, cerramos con unas pequeñas conclusiones acerca de cómo configuramos nuestra natural naturaleza.

6 No entramos aquí en las razones que, lejos de ser ecológicas, sostienen el modelo. 


\section{Los espacios naturales en España}

Nuestro país no tardó en sumarse a la protección de espacios naturales con la declaración del Parque Nacional de la Montaña de Covadonga el 12 de julio de 1918 y el Parque Nacional del Valle de Ordesa el 18 de agosto de ese mismo año. El indiscutible protagonismo, en estas declaratorias, de la figura de $\mathrm{Pe}$ dro Pidal y Bernaldo de Quirós (1870-1941) ha sido múltiples veces señalada (Fernández, 1999 y 2004). No obstante, la primera idea de Parque Nacional parece que se la debemos al ingeniero forestal Rafael Puig i Valls (1845-1920), que propone la creación del Parque Nacional de la Montaña de Montserrat en $1902^{7}$. De hecho, en la propuesta de Puig i Valls encontramos entremezclados los principales componentes regeneradores de la naturaleza: «Fe, ciencia, belleza e identidad nacional» (Casado, 2010: 245).

Más allá de la paternidad atribuida a unos u otros, el desarrollo e implantación de los parques nacionales se debió a una amalgama de factores y raíces. Las preocupaciones regeneracionistas, el higienismo, el desarrollo del naturalismo científico, el excursionismo científico, el alpinismo (pirineísmo), las recreaciones artísticas y literarias del paisaje, las corrientes de renovación pedagógica, el krausismo o el anarquismo naturalista aportaron diferentes bases ideológicas para su desarrollo ${ }^{8}$ (Gómez Mendoza, 1992; Casado, 1997 y 2010; MartíHenneberg, 1994 y 1996; Fernández y Pradas, 2000; Mulero, 2002; Nogué, 2005; Ramos, 2005; Ortega, 2005 y 2007; Castañón y Frochoso, 2007; Morales, 2008; Ortega y García, 2009; García Álvarez, 2009 y 2013). A todas ellas cabe sumar otras raíces fundamentales. De una parte, el trabajo de los ingenieros de montes en la administración forestal. De otra, el gusto de las élites por la cacería y el alpinismo. Esto último supondrá el empuje definitivo del conservacionismo, y será el proteccionismo aristocrático, sintetizado en la figura de Pidal, el responsable de darle forma legal.

En cualquier caso, lo que nos interesa señalar es que todas las instituciones y raíces reseñadas permitieron el descubrimiento de los paisajes de alta montaña y, con ello, una nueva visión de la naturaleza, marcada por las inquietu-

7 El propio contexto catalán favoreció un temprano movimiento conservacionista impulsado por el nacionalismo (Boada, 1995; Boada y Rivera, 2000).

8 Organizaciones como la Institución Libre de Enseñanza, la Sociedad Española de Historia Natural o la Associació Catalanista d'Excursions Científiques fueron fundamentales para su despliegue y condensan las preocupaciones pedagógicas, naturalistas y nacionalistas. 
des regeneracionistas de finales del xix y principios del xx. En este sentido, como señala Nogué para el caso catalán, «la montaña tendrá, cada vez más, un carácter mítico, regenerativo y casi iniciático. Será símbolo de pureza y virginidad» (Nogué, 2005: 155). En este primer movimiento conservacionista, la naturaleza quedaría confinada al imperio vertical de la montaña (Martínez de Pisón, 2000, 2004a y 2004b), y el peso de esta imagen decimonónica seguirá ocupando un lugar destacable en las declaratorias. Así, «el paisaje de montaña periférica, marginal o simbólica, fue, pues, con todas sus limitaciones y tardanzas el primer objetivo de nuestros Parques Nacionales hasta pasada la primera mitad del xx» (Martínez de Pisón, 2007: 24).

Con todo, el crecimiento de las áreas protegidas no se disparó hasta los años noventa del pasado siglo, coincidiendo con la multiplicación de órganos de la Administración estatal, autonómica y local en cuestiones ambientales (Santamarina, 2005a). Tal y como recoge el último informe de Europarc, desde la década de los noventa, el número de áreas naturales «se ha multiplicado por siete, y su superficie se ha triplicado» (2014: 13). Hoy, el $27 \%$ de nuestro país está protegido con alguna figura de conservación de la naturaleza, incluyendo la legislación derivada de nuestra condición europea. En la actualidad, «España alcanza el 12,9 \% de la superficie protegida bajo la figura de espacio natural protegido, superando los siete millones de hectáreas. La superficie marina alcanza las 490000 hectáreas» (Europarc, 2014: 25). Está representada por 1905 lugares protegidos ( 15 parques nacionales, 149 parques naturales, 291 reservas, 328 monumentos, 53 paisajes protegidos y más de 800 espacios con otras figuras de protección). Además, España es el país de la Unión Europea que más superficie terrestre protegida aporta a la Red Natura 2000 (Europarc, 2012 y 2014).

En la Ley 4/1989, de Conservación de los Espacios Naturales y de la Flora y Fauna Silvestres, se definía la figura de parque nacional ${ }^{9}$ del siguiente modo: «Los Parques son áreas naturales, poco transformadas por la explotación $\mathrm{u}$ ocupación humana que, en razón a la belleza de sus paisajes, la representa-

9 La primera definición de parque nacional la encontramos en la Ley de Parques Nacionales de 1916; en ella se establecía que «son parques nacionales, a los efectos de esta ley, los lugares o parajes excepcionalmente pintorescos, boscosos o escabrosos del territorio nacional que el Estado consagra declarándolos así, con el exclusivo objeto de favorecer su accesibilidad por vías de comunicación adecuadas, y de respetar y hacer que se respete la belleza natural de sus paisajes, la riqueza de su fauna y flora y las particularidades geológicas e hidrológicas que contenga, evitando, con la mejor eficacia, cualquier acto de destrucción, deterioro o desfiguración por la mano del hombre» (artículo 2). 
tividad de sus ecosistemas o la singularidad de su flora, de su fauna o de sus formaciones geomorfológicas, poseen unos valores ecológicos, estéticos, educativos y científicos cuya conservación merece una atención preferente» (artículo 13.1). La normativa establecía cuatro figuras de protección nacional: Parques, Reservas Naturales, Monumentos Naturales y Paisajes Protegidos (artículo 4). Con la posterior Ley 42/2007, del Patrimonio Natural y Biodiversidad, la definición se modificaba dando entrada a dos aspectos: el marino, incluyendo las Áreas Marinas, y las prácticas culturales, intentando superar el paradigma dualista (naturaleza/cultura). Estas entradas están en consonancia con las propias transformaciones en la percepción y edificación de los espacios protegidos (Vaccaro, Beltran y Paquet, 2013; Santamarina, Vaccaro y Beltran, 2014) ${ }^{10}$. En esta dirección, se añadía, a las cuatro categorías de protección establecidas, una quinta: Áreas Marinas Protegidas (artículo 29).

Ahora bien, cabe preguntarse, a partir de la aplicación de estas reglamentaciones, cuál es la(s) naturaleza(s) que ha merecido tal distinción. Y es que pese a ser limitados, en los parques nacionales españoles es posible otear qué se ha protegido o protege como natural. En la primera mitad del siglo xx, entre 1918 y 1955, se declaran los seis primeros parques en España: Parque Nacional de la Montaña de Covadonga, el Parque Nacional del Valle de Ordesa, el Parque Nacional del Teide, el Parque Nacional de la Caldera de Taburiente y el Parque Nacional de Aigüestortes i Estany de Sant Maurici. Todos comparten un rasgo en común: son ecosistemas de alta montaña o volcánicos, con altitudes máximas por encima de los 2000 metros, e incluso tres de ellos superan los 3000 metros (Ordesa, Teide y Aigüestortes i Estany de Sant Maurici). Además, los primeros en declararse contienen no solo los elementos rectores del primer conservacionismo (masas boscosas y especies cinegéticas), sino también argumentos patrióticos y nacionalistas ${ }^{11}$.

10 «Tendrán la consideración de espacios naturales protegidos aquellos espacios del territorio nacional, incluidas las aguas continentales y las aguas marítimas bajo soberanía o jurisdicción nacional, incluidas la zona económica exclusiva y la plataforma continental, que cumplan al menos uno de los requisitos siguientes y sean declarados como tales:

a) Contener sistemas o elementos naturales representativos, singulares, frágiles, amenazados o de especial interés ecológico, científico, paisajístico, geológico o educativo.

b) Estar dedicados especialmente a la protección y el mantenimiento de la diversidad biológica, de la geodiversidad y de los recursos naturales y culturales asociados» (artículo 27.1).

11 Véase, entre otros, Fernández (1998), Santamarina (2005), García Álvarez (2009 y 2013), Ortega y García (2009). 
Estos rasgos, sistemas montañosos y volcánicos, dibujados en el primer proteccionismo decimonónico, son los que han seguido primando en nuestro sistema de parques nacionales (tabla 1 ). De hecho, de los quince declarados, diez estarían dentro de estas categorías. Si atendemos a la altitudes máximas, nueve de ellos alcanzan cotas superiores a los mil metros. De este primer atributo podemos concluir que las concepciones de lo natural, o lo representado como el mundo de lo natural, están en ecosistemas terrestres definidos por la altitud, es decir, el lugar de la naturaleza estaría por excelencia en los espacios montañosos, según la visión decimonónica. Así, la verdadera naturaleza se construye de una manera sencilla: es más pura y auténtica cuanto más alta y quebrada sea. Esta mirada está atravesada por lo que podemos denominar la lógica de la transformación. Cuanto más alejados y de más difícil acceso, más naturales se conservarán ciertos territorios, ya que se presuponen menos transformados por el ser humano.

\section{Tabla 1. Parques Nacionales de España por año y superficie}

\begin{tabular}{|c|c|c|c|}
\hline Parque Nacional (Provincia/s) & $\begin{array}{l}\text { Superficie } \\
\text { (ha) }\end{array}$ & $\begin{array}{l}\text { Declaración } \\
\quad \text { (año) }\end{array}$ & $\begin{array}{l}\text { Reclasificación o } \\
\text { ampliación (año) }\end{array}$ \\
\hline $\begin{array}{l}\text { Picos de Europa (Asturias, } \\
\text { Cantabria y León) }\end{array}$ & 64660 & 1918 & 1995 \\
\hline Ordesa y Monte Perdido (Huesca) & 15608 & 1918 & 1982 \\
\hline Teide (Santa Cruz de Tenerife) & 18990 & 1954 & 1981, 1999 \\
\hline $\begin{array}{l}\text { Caldera de Taburiente (Santa Cruz } \\
\text { de Tenerife) }\end{array}$ & 4690 & 1954 & 1981 \\
\hline $\begin{array}{l}\text { Aigüestortes i Estany de Sant } \\
\text { Maurici (Lérida) }\end{array}$ & 14119 & 1955 & 1988,1996 \\
\hline Doñana (Huelva y Sevilla) & 54252 & 1969 & 1978, 2004 \\
\hline $\begin{array}{l}\text { Tablas de Daimiel (Ciudad Real y } \\
\text { Toledo) }\end{array}$ & 1928 & 1973 & 1980 \\
\hline Timanfaya (Las Palmas) & 5107 & 1974 & 1981 \\
\hline Garajonay (Santa Cruz de Tenerife) & 3986 & 1981 & \\
\hline $\begin{array}{l}\text { Archipiélago de Cabrera (Palma de } \\
\text { Mallorca) }\end{array}$ & 10020 & 1991 & \\
\hline Cabañeros (Ciudad Real) & 38996 & 1995 & \\
\hline Sierra Nevada (Granada y Almería) & 86208 & 1999 & \\
\hline
\end{tabular}




\begin{tabular}{|lccc|}
\hline \multicolumn{1}{|c|}{ Parque Nacional (Provincia/s) } & $\begin{array}{c}\text { Superficie } \\
(\mathrm{ha})\end{array}$ & $\begin{array}{c}\text { Declaración } \\
\text { (año) }\end{array}$ & $\begin{array}{c}\text { Reclasificación o } \\
\text { ampliación (año) }\end{array}$ \\
\hline $\begin{array}{l}\text { Islas Atlánticas (Pontevedra y La } \\
\text { Coruña) }\end{array}$ & 8333 & 2002 & \\
\hline Monfragüe (Cáceres) & 17852 & 2007 \\
\hline Guadarrama (Madrid y Segovia) & 33664 & 2013 \\
\hline
\end{tabular}

Fuente: OAPN. Elaboración propia

Hay un segundo rasgo que destacar de nuestro sistema de parques nacionales: cuatro de ellos se sitúan en las islas Canarias, geográficamente ubicadas en África; es, por tanto, la comunidad autónoma canaria la que más parques posee. Además, dos de ellos tienen también el distintivo de Patrimonio de la Humanidad. En este caso, la naturaleza se sitúa en el particular ilusorio occidental, donde la lejanía geográfica configura mundos imaginados y donde el continente africano goza de una naturaleza natural, por su posición geográfica y política.

Y, por último, un tercer rasgo, también apuntado con la declaración de los primeros parques, es el marcado carácter terrestre de los espacios. Hoy, la Red de Parques Nacionales «cubre una superficie de 364626 hectáreas», de la cual el «95\% de la superficie es terrestre» (Europarc, 2014: 28). De los quince que hay, solo hay dos humedales (Doñana y Tablas de Daimiel), y otros dos son mixtos: ecosistemas marino-terrestres (Archipiélago de Cabrera e Islas Atlánticas), lo que sitúa a la naturaleza en un terreno concreto, la tierra firme, frente a otros medios. Esta asociación, como veremos a continuación, responde a la propia historia de la conservación de la naturaleza, dado que la protección del medio marino es muy reciente. De cualquier forma, recordemos que, en el Estado español, la figura de Área Marina Protegida (AMP), como una categoría más de las áreas naturales, aparece con la Ley 42/2007 antes referida. En esa ley se definen como «espacios naturales designados para la protección de ecosistemas, comunidades o elementos biológicos o geológicos del medio marino, que, en razón de su rareza, fragilidad, importancia o singularidad, merecen una protección especial» (artículo 31.1). Será a partir de 2010 cuando se constituya la Red de Áreas Marinas Protegidas de España (RAMPE) (Ley 41/2010), con el propósito de impulsar la protección del medio marino. La superficie marina 
protegida en 2014 alcanzaba las 490000 hectáreas (Europarc, 2014) con un área marina protegida (El Cachucho).

En suma, la idea de lo natural que se desprende de la Red de Parques Nacionales de España es que estos son en su mayoría terrestres, se sitúan lejos, en lo real y en lo simbólico, tanto por la altitud como por su posición geográfica. La montaña agreste y los espacios en el continente africano sustentan la mayoría de nuestra red. Y, como ya ha sido puesto en evidencia, «esta red es producto más de la historia que de la geografía» y no responde a «un plan y una selección coherente del territorio susceptible de conservación» (Martínez de Pisón, 2007: 25). Las razones ecológicas parecen quedar a merced de las razones político-económicas.

\section{La patrimonialización natural de la Unesco}

La patrimonialización de la naturaleza global ha sufrido, en los últimos años, un crecimiento espectacular. Pese a que el origen del conservacionismo hay que situarlo en el xix, con la declaración de los primeros parques nacionales del mundo, no será hasta la década de los ochenta del pasado siglo cuando arranque una conservación, cualitativa y cuantitativamente, expansiva y continuada, movimiento que sigue creciendo, tal y como señala el último informe Protected Planet Report, «Over the past 20 years, there has been a dramatic increase in the number and extent of protected areas established globally» (UNEP-WCMC y IUCN, 2016: 10). Hoy, según señala esta misma fuente, «There are 202467 terrestrial and inland water protected areas recorded in the World Database on Protected Areas (WDPA), covering $14.7 \%$ (19.8 million $\left.\mathrm{km}^{2}\right)$ of the world's extent of these ecosystems (excluding Antarctica)» (UNEP-WCMC y IUCN, 2016: 30).

El crecimiento de los espacios protegidos se contextualiza en un periodo donde proliferan organismos e instituciones que se consagran a la protección y conservación de la naturaleza, por la propia coyuntura de la crisis ecológica global. La Unión Internacional para la Conservación de la Naturaleza (UICN) se ha constituido como la corporación internacional más prestigiosa para este fin. De hecho, ha realizado un extenso trabajo normativo definiendo categorías 
para el manejo de las áreas protegidas ${ }^{12}$. Desde su perspectiva, un área protegida es: «An area of land and/or sea especially dedicated to the protection and maintenance of biological diversity, and of natural and associated cultural resources, and managed through legal or other effective means». Junto a la UICN, el programa MAB (Hombre y Biosfera) promovido por la Unesco ha impulsado la creación de una red internacional de espacios protegidos con el rótulo de Reservas de la Biosfera ${ }^{13}$.

La propia Unesco, a través de su programa de protección del Patrimonio de la Humanidad y apoyada por la UICN, en la Convención sobre la Protección del Patrimonio Mundial, Cultural y Natural de París en 1972, estableció la distinción de Patrimonio Cultural y Natural de la Humanidad. Con esta convención asistimos a la globalización del patrimonio, con un giro copernicano en su concepción (Ariño, 2002; Kirshenblatt-Gimblett, 2004; Santamarina, 2005b). De esta forma, la Unesco instituyó que existían bienes que debían ser salvaguardados y conservados, porque eran patrimonio común de la humanidad. Por tanto, se debía velar por los mismos pese a que los países portadores no los custodiaran. Para ello se creó el Comité del Patrimonio Mundial, encargado de considerar las candidaturas presentadas y asesorados por el ICOMOS, el ICCROM y la UICN. Para ser incluidos en la lista, los bienes, culturales y naturales, se seleccionarían a partir de unos criterios establecidos por la institución.

A la hora de definir los bienes naturales, la Unesco utilizó un patrón similar al del patrimonio cultural, aunque, en este caso, los criterios fueron cuatro frente a los seis del cultural: a) que fueran ejemplos sobresalientes y representativos de los diferentes periodos de la historia de la Tierra; $b$ ) que fueran representativos de la evolución biológica; $c$ ) que contuvieran fenómenos naturales extraordinarios o áreas de una belleza o estética excepcional; y d) que albergaran

12 En total define seis: Reserva Natural Estricta / Área Natural Silvestre: Protección estricta con fines científicos / fines de protección de la naturaleza salvaje; Parque Nacional: ecosistema para conservación y recreo; Monumento Natural: conservación de características naturales específicas; Área Ordenación de Hábitat / Especies: conservación y gestión a través de una ordenación activa; Paisaje Marítimo-Terrestre Protegido: conservación y recreo; y Área Protegida de Ordenación de Recursos: ecosistemas naturales para uso sostenible.

13 No vamos a tratar aquí todos los organismos o tratados de protección de la naturaleza. Solo añadiremos que existen otros, como el Convenio de Ramsar (1975), para proteger zonas de humedales o, en nuestro contexto, la Directiva Hábitats de la Unión Europea (1992), que perseguía crear una red de espacios naturales protegidos europeos (Red Natura 2000). 
hábitats naturales representativos para la conservación in situ de la diversidad biológica, incluyendo las especies amenazadas. Los bienes naturales se clasificaron a partir de tres categorías: Monumentos naturales; Formaciones físicas y biológicas con valor universal estético o científico (volcanes, cataratas, barrancos, etc.); Formaciones geológicas y fisiográficas que constituyan el hábitat de especies amenazadas (formaciones marinas, albuferas, selvas, desiertos, etc.); Lugares naturales o zonas naturales delimitadas, que tengan un valor universal excepcional desde el punto de vista científico y estético (Amazonas, Antártida, parques naturales, etc.).

El trabajo normativo realizado para clasificar y ordenar el patrimonio natural y cultural como Patrimonio de la Humanidad, por un lado, fortalecía la dicotomía naturaleza/cultura y, por otro, bendecía el modelo de los parques nacionales al darles, mayoritariamente, como veremos a continuación, la nueva distinción creada. Pero analicemos qué se dibuja detrás de estas declaraciones.

Los primeros espacios naturales como Patrimonio Mundial fueron declarados en $1978^{14}$, cuando se activó dicha lista con la proclamación de doce bienes Patrimonio de la Humanidad. Cuatro de ellos fueron naturales (Parque Nacional del Nahanni, Islas Galápagos, Parque Nacional de Yellowstone y Parque Nacional de Simien) (tabla 2). Esta primera declaratoria ya apuntaba, por un lado, en la dirección de cómo se irían tejiendo los mapas patrimoniales de la Unesco no solo por el desequilibrio de sus listas, sino por su distinta ubicación y desigualdad en el reparto de bienes (Santamarina, 2013). Y, por otro lado, comenzaba a indicar dónde y cómo se situaba la natural naturaleza. Tres de los espacios se ubicaban en América y el otro en África, los cuatro con una altitud considerable y con relieve montañoso o volcánico. El ecosistema dominante era el terrestre, pese a que se identificaba a las islas Galápagos como ecosistema mixto. Respecto a estas islas es destacable su particular relación con el naturalista por excelencia y precursor de la ecología, Darwin, y su condición, por tanto, de encapsuladoras de mitos prístinos y de referentes ecológicos. En esta línea, se reconocía también, y como no podría ser de otro modo, al Parque Nacional de Yellowstone, el precursor y modelo de la expansión de los espacios naturales. De esta manera, podemos ver que la primera declaración contenía, más allá de criterios biológicos conservacionistas, una carga ideológica de peso.

14 En 1978 se activará la Lista del Patrimonio Mundial Cultural y Natural, con la declaración de doce bienes patrimonio de la humanidad de todo el mundo. 
Tabla 2. Primeros sitios declarados Patrimonio Natural de la Humanidad

\begin{tabular}{|l|c|c|c|c|}
\hline \multicolumn{1}{|c|}{ Nombre del bien } & País & Continente & Ecosistema & $\begin{array}{c}\text { Altura } \\
\text { máxima }\end{array}$ \\
\hline Parque Nacional Nahanni & Canadá & América & Terrestre & $2640 \mathrm{~m}$ \\
\hline Islas Galápagos & Ecuador & América & Terrestre / Acuático & $1690 \mathrm{~m}$ \\
\hline $\begin{array}{l}\text { Parque Nacional } \\
\text { de Yellowstone }\end{array}$ & Estados Unidos & América & Terrestre & $3462 \mathrm{~m}$ \\
\hline Parque Nacional de Simien & Etiopía & África & Terrestre & $4553 \mathrm{~m}$ \\
\hline
\end{tabular}

Fuente: Unesco. Elaboración propia

$\mathrm{Si}$ atendemos a los primeros años, por ejemplo al primer quinquenio, podemos observar cómo se dibuja la tendencia que acabamos de apuntar. Con los años ha ido perdiendo fuerza, pero es significativa en la medida que indica qué se consideraba prioritario para conservar, un orden, ranking o jerarquía de lugares de la naturaleza que se consideraban más naturales ${ }^{15}$. Lo primero que llama la atención, en el primer periodo, es que el continente americano y africano coparon el $78 \%$ de la lista, frente al $12 \%$ asiático, el $6 \%$ europeo o el $3 \%$ oceánico (gráfico 1). La segunda característica destacable es que el $77 \%$ de los espacios declarados eran ya parques nacionales en sus respectivos países. La tercera peculiaridad nos lleva a la altura: el $68 \%$ de los espacios superaba los 1000 metros de altitud (en concreto, el $42 \%$ rebasaba los 2000 metros, y el $16 \%$, los 4000). Y, por último, el $63 \%$ de los ecosistemas eran terrestres y solo dos eran acuáticos (costeros-marinos).

15 Recordemos que hablamos de patrimonio de la bumanidad, de un ejercicio metacultural, según palabras de Kirshenblatt-Gimblett (2004), que, en nuestro caso, siguiendo su lógica, sería metanatural. 


\section{Gráfico1. Distribución del Patrimonio Natural de la Humanidad por continentes geográficos en el primer quinquenio}

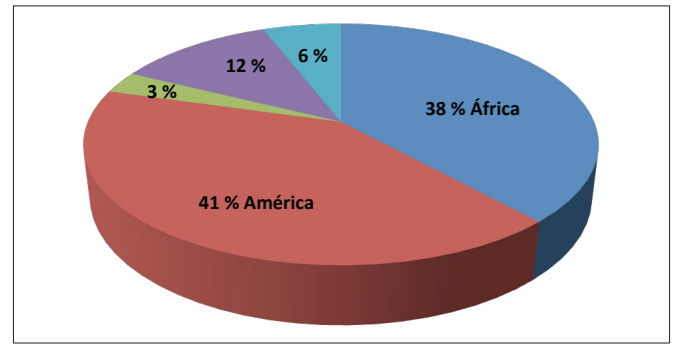

Fuente: Unesco. Elaboración propia

En síntesis, estos primeros años de declaratorias estuvieron marcados por una ubicación de lo natural bien definida. De las particularidades señaladas resaltamos dos. Por una parte, el hecho de que se primaran espacios ya declarados, como los parques nacionales. Una tendencia que ha continuado, ya que aproximadamente el $50 \%$ de su lista se nutre de ellos. Con esta política se respaldaba la creación de áreas naturales protegidas como vía para la conservación, sin cuestionarlas, reforzando el viejo proteccionismo decimonónico. Al mismo tiempo se empezaban a duplicar las distinciones al otorgar a un mismo espacio distintas protecciones. Esto no solo complicaría el manejo de un espacio, sino que cuestionaría el sentido de proteger lo que ya está protegido, lo que obliga a preguntarse sobre la necesidad real, o sobre la estrategia que hay detrás, de acumular distinciones. No está de más señalar que el resto de las áreas naturales de la lista tienen también otras figuras de protección asociadas (Reserva de la Biosfera, Parque Provincial, Zona de Conservación, Parque Marino, Santuario, etcétera). Por otra parte, insistimos en la localización geográfica de los bienes naturales centrada en dos continentes. El Nuevo Mundo, el adjetivo ya es indicativo, y África, el territorio más primitivo y salvaje en el imaginario occidental, representan, de manera casi perfecta, nuestra consideración de lo natural.

A día de hoy, en la Lista de Patrimonio de la Humanidad hay declarados 203 bienes naturales. Si los comparamos con los bienes culturales observaríamos un claro desequilibrio, dado que estos cuadruplican su presencia. No podemos compararlos con la lista de bienes inmateriales, que en 2015 contenía 
348 bienes declarados, porque esta lista de patrimonio inmaterial es muy joven (se activa en el año 2008). De igual manera sucede si prestamos atención a su emplazamiento: la Unesco proyecta una clara geopolítica de dónde se encuentra lo natural en su distribución geográfica. Si atendemos al número total de bienes declarados, el continente americano es el que más peso tiene en el total mundial, seguido del continente africano y el gigante asiático (véase gráfico 2).

\section{Gráfico 2. Distribución del Patrimonio Natural de la Humanidad por continentes geográficos}

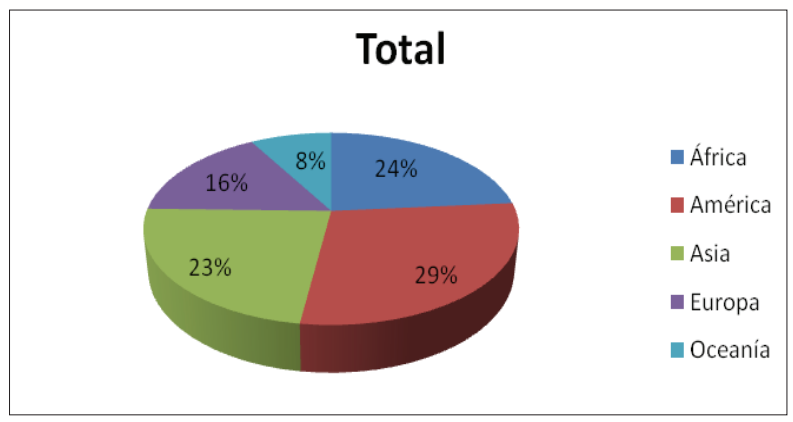

Fuente: Unesco. Elaboración propia

Si prestamos ahora atención a la distribución americana, observaremos que la mayoría de los bienes se concentran en tres países: Estados Unidos (12), Canadá (9) y Brasil (7). Estos tres países aglutinan casi la mitad de todos los sitios declarados de patrimonio natural de América y vale la pena demorarse en ellos para observar los rasgos que los definen. En concreto, podemos ver que los espacios naturales ubicados en países del hemisferio norte se caracterizan por ser terrestres (todos salvo uno, que es terrestre/acuático), por su carácter montañoso y por su altitud (en Estados Unidos el 83 \% está por encima de los 2000 metros, en concreto, cinco de ellos alcanzan los 4000 metros o más), mientras que los situados en el hemisferio sur vienen representados mayoritariamente por la selva amazónica, lo que implica la entrada de otras consideraciones. En el caso de Brasil, seis de sus bienes se encuentran clasificados como ecosistemas de selva tropical, cinco de ellos están por debajo de los 500 metros y solo uno alcanza una altitud de más de 1000 metros. En su mayoría siguen siendo terrestres, aunque hay dos clasificados como terrestres/acuáticos. ¿Qué 
conclusiones podemos sacar de este pequeño tanteo? Sin duda, que el relieve de alta montaña y la selva son dos imágenes contundentes en la representación de la naturaleza. Ambas se encuentran, en nuestro sistema de cognición, lejanas e impracticables. Y esa lejanía, real o simbólica, a la vez que histórica y geográfica, las hace aparecer como espacios naturales por excelencia.

En suma, y para ir cerrando esta pequeña incursión, desde nuestro punto de vista, lo terrestre, la distancia y la altitud, junto con algún ecosistema en particular (selva), marcan la lista de una forma clara. Si atendemos al número de bienes que se sitúan por encima de los 500 metros, en el total de la lista representan el $70 \%$. El otro $30 \%$ se reparte entre zonas de selva y jungla, y ecosistemas marinos, costeros o humedales. Del mismo modo, podemos ver que los ecosistemas terrestres ocupan la mayoría de los sitios declarados (gráficos 3 y 4). Si prestamos atención a las fechas de las distintas declaraciones, veremos que en la década de los ochenta solo se declaran tres ecosistemas acuáticos (uno de ellos un lago de montaña), lo que, como ya hemos indicado, es revelador de dónde se encuentra la naturaleza. En realidad, esto está en consonancia con la propia historia de la conservación de las áreas marinas protegidas (AMP). Esta protección ha sido muy posterior a la terrestre. Pese a que se inicia de manera tímida en la década de 1970, no despega hasta el año 2000. Todavía hoy, cualitativa, cuantitativa y proporcionalmente (teniendo en cuenta el reparto de la superficie de la tierra), es mucho menor su custodia. De forma aproximada, ocupa la mitad de kilómetros cuadrados que la terrestre. En 2011, según la base de datos de MPA Global, se protegía una superficie global de 24236 478,69, de las cuales 16263 609,39 eran terrestres y 8106 430,34 eran marinas ${ }^{16}$. Es decir, las AMP representaban una superficie de menos del $1 \%$ de todos los ecosistemas marinos del mundo. Cinco años después, como se indica en Protected Planet Report, se ha producido un enorme crecimiento de las AMP, con el aumento de la protección en un 1,8 \% (equivalente a 2,6 millones de $\mathrm{km}^{2}$ ). En 2016 «there are 14688 Marine Protected Areas (MPA) recorded in the World Database on Protected Areas (WDPA), covering 4.12 \% (14.9 million $\mathrm{km}^{2}$ ) of the global ocean and $10.2 \%$ of coastal and marine areas under national jurisdiction» (UNEP-WCMC y IUCN, 2016: 32).

16 Véanse datos en $<$ http://www.wdpa.org $>$. 
Gráfico 3. Distribución del Patrimonio Natural de la Humanidad por ecosistema

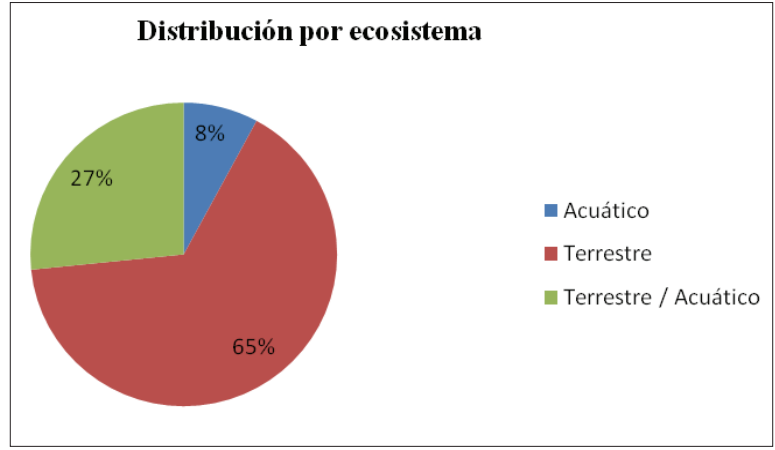

Fuente: Unesco. Elaboración propia

Gráfico 4. Distribución del Patrimonio Natural de la Humanidad por ecosistema y continente

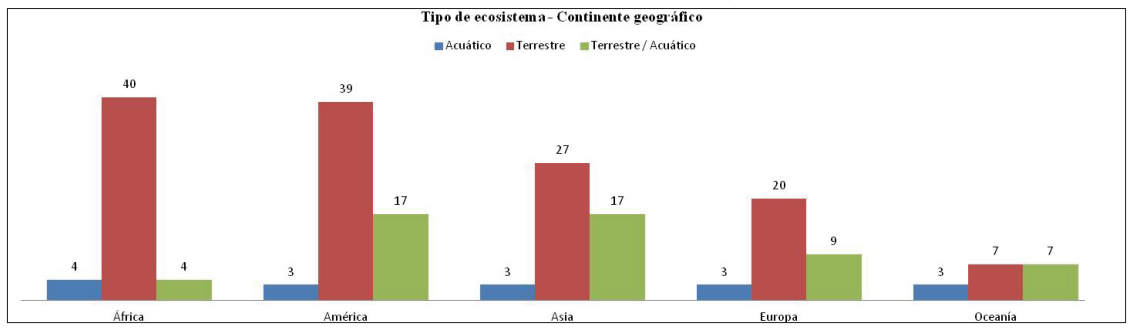

Fuente: Unesco. Elaboración propia

\section{Conclusiones}

Podemos decir que hoy la filosofía del wilderness sigue vigente y se ampara en la necesidad de seguir construyendo áreas protegidas naturales, para disfrutar de una naturaleza intemporal y conservar así para el futuro un pasado tan mitificado como intervenido. Desde los primeros parques hasta nuestros días, el imaginario occidental no ha hecho más que engrandecer esta fantasía (Igoe, 2006). El éxito es tal que hoy la natural naturaleza se ha convertido en todo un mercado creciente, que disfruta de una alta demanda, produciendo una suerte de naturaleza mercantilizada que garantizaría paradójicamente su conservación, a la vez que su conservación garantizaría los circuitos de 
su distribución (Beltran, Pascual y Vaccaro, 2008; Vaccaro y Beltran, 2007; Igoe, Neves y Brockington, 2010; Brockington y Duffy, 2010; Buscher et alii, 2012). Lo natural se ha transformado en una potente marca distintiva para espacios, productos, localidades y personas (Santamarina y Bodí, 2013), y se «ha espectacularizado» la naturaleza idealizada (Buscher et alii, 2012). La naturaleza salvaje, la verdadera y la genuina, sigue siendo un auténtico motor ideológico que conforma un discurso hegemónico global sobe la conservación (Holmes, 2011) y configura mapas políticos de intervención.

Ahora bien, es importante anotar que esta manera de visionar lo natural tiene importantes consecuencias políticas, al condicionar nuestra manera de desenvolvernos en nuestro ambiente. Siguiendo con el análisis efectuado en este trabajo, la clasificación de lugares como naturales o no, así como su gradación, su emplazamiento y sus características, determinan nuestras relaciones con el medio. El hecho de alejarlos (en el espacio y en un tiempo mítico) o de no reconocerlos (por la lejanía señalada) conlleva el riesgo de percibir su degradación como algo desplazado en el tiempo y en el espacio, lo que dificultaría una visión interrelacionada y una conservación efectiva. Para finalizar, resta decir que al imponer un modelo taxonómico global de la naturaleza — una jerarquización normalizada de valores naturales - a través de instituciones globalizadas y hegemónicas, otras naturalezas se pierden. La construcción de una metanaturaleza responde a criterios occidentales (globocéntricos), que borran otras posibles naturalezas, y con ellas, otras formas de relacionarnos en nuestro mundo. En definitiva, «si es cierto que siempre hay formas de posdesarrollo, no capitalismo y "otras naturalezas" en construcción, entonces hay esperanza de que se puedan llegar a constituir nuevas bases para la existencia y rearticulaciones significativas de la subjetividad y de la alteridad en sus dimensiones económica, cultural y ecológica» (Escobar, 2000: 209). 


\section{Bibliografía}

Adams, W.y Hutton, J. (2007). «People, Parks and Poverty: Political Ecology and Biodiversity Conservation», Conservation and Society, 5 (2): 147-183.

Ariño, A. (2002). «La expansión del patrimonio cultural», Revista de Occidente, 250: 129-150.

Beltran, O; Pascual, J. y Vaccaro, I., (coord.) (2008). Patrimonialización de la naturaleza. El marco social de las políticas ambientales. Donosti: Ankulegi.

Boada, M. (1995). Rafael Puig i Valls (1845-1920). Precursor de l'educació ambiental $i$ dels espais naturals protegits. Barcelona: Generalitat de Catalunya.

Boada, M.y Rivera, M. (2000). «L'origen dels espais naturals protegits», Medi ambient: Tecnología i cultura, (27), 5-13.

Bourdieu, P. (1991). El sentido práctico. Madrid: Taurus

Bourdieu, P. (1998). La distinción. Criterio y bases sociales del gusto. Madrid: Taurus.

Brockington, D. y Duffy, R. (2010). «Capitalism and Conservation. The production and reproduction of biodiversity conservation», Antipode 42(3): 469-84.

Brockington, D.; Duffy, R. y Igoe, J.(2008). Nature Unbound. Conservation, Capitalism and the Future of Protected Areas. Londres: Earthscan.

Buscher, B.; Sullivan, S.; Neves, K.; Igoe, J. y Brockington, D. (2012). «Towards a synthesized critique of neoliberal biodiversity conservation», Capitalism Nature Socialism 23 (2): 4-30.

Casado de Otaola, S. (2010). Naturaleza Patria. Ciencia y sentimiento de la naturaleza en la España del regeneracionismo. Madrid: Fundación Jorge Juan-Marcial Pons.

Casado de Otaola, S. (1997). Los primeros pasos de la ecología en España. Madrid: Ministerio de Agricultura, Pesca y Alimentación.

Castañón, J. C. y Frochoso, M. (2007). «La naturaleza del paisaje en el Parque Nacional de los Picos de Europa». En Martínez de Pisón, E. y Ortega, N. (eds.): La conservación del paisaje en los Parques Nacionales. Madrid: Universidad Autónoma de Madrid y Fundación Duques de Soria. 177-212. 
Comaroff, J. y Comaroff, J. (1991). Of revelation and revolution. Chicago: University of Chicago Press.

Daniels, S. (1993). Fields of vision: Landscape Imaginary and National Identity in England and the United States. Princeton: Princeton University Press.

Descola, P. (2007). «Posface. Les coulisses de la natura», Cabiers d'antropologie sociale, $3: 123-127$.

Descola, P. y Pálsson, G. (eds.) (1996). Nature and Society. Anthropological perspectives. Londres: Routledge.

Eder, K. (1996). The Social Construction of Nature. A Sociology of Ecological Enlightenment. Londres: Sage.

Escoвar, A. (1998). «Whose knowledge, whose nature? Biodiversity, conservation, and the political ecology of social movements», Journal of Political Ecology 5, 53-82.

- (2000). «El lugar de la naturaleza y la naturaleza del lugar». En A. Viola, (comp.): Antropología del desarrollo. Barcelona: Paidós

Europarc (2012). Anuario Europarc-España del estado de los espacios naturales protegidos 2011. Madrid: Fundación Fernando González-BBVA.

- (2014). Anuario Europarc-España del estado de los espacios naturales protegidos 2013. Madrid: Fundación Fernando González-BBVA.

Fernández, J. (1998). El hombre de Picos de Europa. Pedro Pidal, marqués de Villaviciosa: fundador de los Parques Nacionales. Madrid: Caja Madrid.

Fernández, J. y Pradas, R. (2000). Los Parques Nacionales Españoles (Una aproximación bistórica). Madrid: Organismo Autónomo de Parques Nacionales.

Fortunato, N. (2005). «El territorio y sus representaciones como recurso turístico», Estudios y perspectivas en turismo, 14: 314-348.

Foucault, M. (1970). The Order of Things: An Archaeology of the Human Sciences, London: Tavistock.

- (1991). Saber y verdad. Madrid: La Piqueta.

García Áltvarez, J. (2009). «Lugares, paisajes y políticas de memoria: una lectura geográfica», Boletín de la A.G.E., 51: 175-202.

- (2013). «Paisajes, memoria histórica e identidad nacional en los inicios de la política de conservación de la naturaleza en España: de Covadonga a San Juan de la Peña», Hispania, 73, (244): 409-438. 
Gómez, J. (1992). Ciencia y política de los montes españoles (1848-1936). Madrid: ICONA.

Haraway, D. (1995). Ciencia, cyborgs y mujeres. La reinvención de la naturaleza. Madrid: Cátedra.

Holmes, G. (2011). «Conservation's Friends in High Places: Neoliberalism, Networks, and the Transnational Conservation Elite», Global Environmental Politics, 11(4): 1-21.

Hornborg, A. y Pálsson, G. (2000). Negotiating Nature: Culture, Power, and Environmental Argument. Lund: Lund University Press.

Hutton, J.; Adams, W. y Murombedzi, J. C. (2005). «Back to the Barriers? Changing Narratives in Biodiversity Conservation», Forum for Development Studies, 2: 341-370.

Igoe, J. (2006). «Measuring the Costs and Benefits of Conservation to Local Communities», Journal of Ecological Anthropology, 10: 72-77.

Igoe, J; Neves, K. y Brockington D. (2010). «A Spectacular Eco-Tour around the Historic Bloc: Theorising the Convergence of Biodiversity Conservation and Capitalist Expansion», Antipode, 42(3): 486-512.

Kirshenblatt-Gimblett, B. (2004). «El patrimonio inmaterial como producción metacultural», Museum International, 221: 52-67.

Latour, B. (1993). Nunca hemos sido modernos. Ensayo de antropología simétrica. Madrid: Debate.

Martí Henneberg, J. (1994). Lexcursionisme cientific i la seva contribució a les ciències naturals $i$ a la geografia. Barcelona: Alta Fulla.

- (1996). «El excursionismo científico», Mundo científico, 173: 962-969.

Martínez de Pisón, E. (2000). «Imagen de la naturaleza de las montañas». En Martínez de Pisón, E. y Sanz Herräiz, C. (eds.): Estudios sobre el paisaje. Madrid: Universidad Autónoma de Madrid y Fundación Duques de Soria, pp. 15-54.

Martínez de Pisón, E. (2004b). «Valores escondidos de los paisajes. Calidades ocultas de la ascensión a la montaña». En Martínez de Pisón, E. y OrTEgA, N. (eds.): Los valores del paisaje. Madrid: Universidad Autónoma de Madrid y Fundación Duques de Soria, pp. 9-44.

Martínez de Pisón, E. (2004a). «El paisaje de montaña. La formación de un canon natural del paisajismo moderno». En Ortega Cantero, N. (ed.): 
Naturaleza y cultura del paisaje. Madrid: Universidad Autónoma de Madrid y Fundación Duques de Soria. 15-53.

Martínez de Pisón, E. (2007). «Reflexión geográfica sobre los paisajes y los Parques Nacionales». En Martínez de Pisón, E. y Ortega, N. (eds.): La conservación del paisaje en los Parques Nacionales. Madrid: Universidad Autónoma de Madrid y Fundación Duques de Soria. 9-36.

Mignolo, W. (2012). Local histories/global designs: Coloniality, subaltern knowledges, and border thinking. Princeton: Princeton University Press.

Morales, A. (2008). «Historia, literatura, paisaje». En Martínez de Pisón, E. y Ortega, N. (eds.): La recuperación del paisaje. Madrid: Universidad Autónoma de Madrid-Fundación Duques de Soria, pp. 65-86.

Mulero, A. (2002). La protección de los espacios naturales en España. Madrid: Mundi-Prensa.

Nash, R. (1970). «The American Invention of National Park», American Quarterly, 22 (3): 726-735.

Nogué, Joan (2005). «Nacionalismo, territorio y paisaje en Cataluña». En Ortega, N. (ed.): Paisaje, memoria bistórica e identidad nacional. Madrid: Universidad Autónoma de Madrid-Fundación Duques de Soria, pp. 146169.

Ortega Cantero, N. y García Álvarez, J. (2009). «Paisaje y lugares de memoria: Covadonga y El Paular». En Martínez de Pisón, E. y Ortega Cantero, N. (eds.): Los valores del paisaje. Madrid: Ediciones de la Universidad Autónoma de Madrid Fundación Duques de Soria, pp. 45-93.

Ortega Cantero, N. (2007). «El significado cultural del Parque Nacional del Guadarrama». En Martínez de Pisón, E. y Ortega, N. (eds.): La conservación del paisaje en los Parques Nacionales. Madrid: Universidad Autónoma de Madrid y Fundación Duques de Soria, pp. 65-98.

Ortega Cantero, N. (ed.) (2005). Paisaje, memoria histórica e identidad nacional. Madrid: Universidad Autónoma de Madrid-Fundación Duques de Soria.

Ramos Gorostiza, J. L. (2005). «Concepciones económicas: en los inicios de la conservación de la naturaleza en España: nexos y contrastes con el caso estadounidense», Revista de Historia Industrial, 28: 11-45.

Runte, A. (1977). "The National Park Idea: Origins and Paradox of the American Experience», Journal of Forest History, 21 (2): 64-75. 
Santamarina, B. (2005a). «La patrimonialización de la naturaleza: figuras y discursos». En Pascual, J. y Florido, D. (eds.): ¿Protegiendo los recursos? Áreas protegidas, poblaciones locales y sostenibilidad, 9-44. Sevilla: Fundación Monte.

- (2005b). «Una aproximación al patrimonio cultural». En Hernández, G; Santamarina, B.; Moncusí, A. y Albert, M.: La memoria construida. Patrimonio cultural y modernidad. Valencia: Tirant Lo Blanch. 21-51.

- (2009). « DeParques y Naturalezas. Enunciados, Cimientos y Dispositivos», Revista de Dialectología y Tradiciones Populares, 64(1): 297-324.

- (2013). «Los mapas geopolíticos de la Unesco: entre la distinción y la diferencia están las asimetrías. El éxito (exótico) del patrimonio inmaterial», Revista de Antropología Social. UCM.

Santamarina, B. y Bodí, J. (2013). «Lugares rurales versus espacios naturalizados. Conocimientos y reconocimientos en las lógicas patrimoniales de las áreas protegidas», AIBR, Revista de Antropología Iberoamericana, 8(1), 111-138.

Santamarina, B.; Vaccaro, I. y Beltran, O. (2014). «La patrimonialización de la naturaleza: génesis, transformaciones y estado actual», Arxius de Ciències Socials, 30: 87-98.

Selmi, A. y Hirtzel, V (2007), «Gouverner la nature», Cabiers d'antropologie sociale, (3):9-12.

Smith, N. (2007). «Nature as accumulation strategy», Socialist register, 2007: 19-49

Solé, J·y Bretón, V. (1986). «El paraíso poseído. La política española de los parques naturales (1880-1935)», Geocrítica, 63: 1-59.

Stevens, S. (1997). «The legacy of Yellowstone». En Stevens, S. (ed.): Conservation through cultural survival: indigenous people and protected areas. Washington: Island Press. 13-32.

UNEP-WCMC y IUCN (2016). Protected Planet Report 2016. Cambridge y Gland: UNEP-WCMC and IUCN.

Vaccaro, I. y Beltran, O. (eds.) (2007). Ecología política de los Pirineos. Estado, bistoria y paisaje. Barcelona: Garsineu Edicions.

Vaccaro, I.; Beltran, O. y Paquet, P. (2013). «Political ecology and conservation policies: some theoretical genealogies» Journal of Political Ecology, 20: 255-272. 
West, P. y Brockington, D. (2006). «An Anthropological Perspective on Some Unexpected Consequences of Protected Areas», Conservation Biology, 20(3): 609-616.

West, P.; Igoe, J. y Brockington, D. (2006). «Parks and peoples: The social impact of protected areas», Annual Review of Anthropology, 35: 251-277. 